\title{
Sixteenth Annual Saskatchewan Christmas Bird Count, 1957
}

\author{
By Mary Houston, Yorkton, Sask.
}

This year, Christmas bird count reports were received from 20 localities in Saskatchewan. The total number of species seen on "Count Day" for all localities was 47 , with an additional 4 species seen during Christmas week but not on the day on which the official counts were taken.

From this year's report, 3 new species were added to the master list of birds seen in Saskatchewan during Christmas week in the last 16 years. This brings the total number of: species to 88 . New this year were: American Widgeon (Baldpate) Blue-winged Teal, and Rusty Blackbird.

Of the 20 localities, 19 reported seeing Black-billed Magpies, 18 Blackcapped Chickadees, 17 Common Redpolls, 16 House Sparrows, 14 Pine Grosbeaks, 12 Hairy Woodpeckers, and Downy Woodpeckers, 11 Sharptailed Grouse and Snow Buntings. It has been a good year for Common Redpolls and Pine Grosbeaks, both of which have been seen in more areas than previously.

The fact that there was little snow in most areas made travelling about easier. Worthy of special mention are:

(a) The enthusiasm of Don Karasiuk, the 11 year old Prince Albert boy who sent in a very professional report.

(b) The count made in the Qu'Appelle Valley at Craven by members of the Museum staff. They also saw 48 individuals of 8 species of mammals.

(c) Steve Mann of Skull Creek, who got together the third largest group of bird counters.

(d) The Regina group who, led by Elmer Fox, compiled the largest number of species-24 in one day. (e) The Saskatoon Natural History Society who got out the largest number of counters, scoured the area thoroughly, and turned in the largest number of individual birds seen in any locality this year.

BIG RIVER, Decmeber 26, $4 \mathrm{mi}$. on foot in 3 hours; temp. $8^{\circ}$; wind $\mathrm{NE}$ 20 mph; snowing; 12 inches of snow en ground. 12 species, 86 individuals. Sharp-tailed Grouse, 3; Gray (Hun- garian) Partridge, 4; Great Horned Owl, 1; Pileated Woodpecker, 1; Hairy Woodpecker, 4; Downy Woodpecker, 2; Blue Jay, 7; Black-billed Magpie, 3; Common Raven, 3; Blackcapped Chickadee, 32; Common Redpoll, 11; Snow Bunting, 15. (Add: White-breasted Nuthatch, 1; Evening Grosbeak, 7; Pine Grosbeak, 3.) Mrs. Anne Olson.

BLADWORTH, December 26, $14 \mathrm{mi}$. in $4 \mathrm{hrs}$. by team and truck; temp. 18 to $34^{\circ}$; wind $\mathrm{SE}$ at $20 \mathrm{mph}$; cloudy; 4-5 inches of snow. 8 species, 211 individuals. Sharp-tailed Grouse, 8; Gray (Hungarian) Partridge, 13; Great Horned Owl, 1; Blue Jay, 2; Black-billed Magpie, 5; Black-capped Chickadee, 1; House Sparrow, 30; Common Redpoll, 151. (Add: Snowy Owl, 1, Dec. 31; Downy Woodpecker, 1, Dec. 24; Horned Lark, 1, Dec. 27; Bohemian Waxwing, 37 on Dec 24; Northern Shrike, 1, Dec. 24; Snow Bunting, 36, Dec. 27.) - P. Lawrence Beckie.

CARLTON, December 29, $2 \mathrm{mi}$. on foot $67 \mathrm{mi}$. by car, $1 \frac{1 / 2}{2} \mathrm{hrs}$. on foot, 13 hrs. by car; temp. -34 to $-25^{\circ}$; wind $\mathrm{E}$ and $\mathrm{NE}$ at $15 \mathrm{mph}$.; mostly overcast; 6 in. of snow. 15 species, 688 individuals. Pigeon Hawk, 1; Ruffed Grouse, 5; Sharp-tailed Grouse, 18; Gray (Hungarian) Partridge, 16; Snowy Owl, 1; Hairy Woodpecker, 1; Gray (Canadia) Jay, 1; Black-billed Magpie, 19; Blackcapped Chickadee, 10; Starling, 5; House Sparrow, 168; Pine Grosbeak, 19; Evening Grosbeak, 22; Common Redpoll, 151; Snow Bunting, 251. -

Margaret Belcher (Regina Natural History Society); Dr. R. M. Bremner, Jonathon Gerrard, J. Frank Roy (Saskatoon Natural History Society); Dir. and Mrs. Stuart Houston (compilers, (Yorkton Natural History Society).

CRAVEN, December $30,4 \mathrm{mi}$. on foot, $39 \mathrm{mi}$. by car, in $7 \frac{1}{1} \mathrm{hrs}$.; temp. -17 to $-2^{\circ}$ sunny; winds southerly, 6 mph.; 19 species, 779 individuals. Golden Eagle, 2; Bald Eagle, 2; Ruffed Grouse, 5; Sharp-tailed Grouse, 2; Great Horned Owl, 1; Snowy Owl, 1; Short-eared Owl, 1; Hairy Woodpecker, 9; Downy Woodpecker, 9; 
Horned Lark, 1; Black-billed Magpie, 44; Black-capped Chickadee, 27; Bohemian Waxwing, 35; Northern Shrike, 1; House Sparrow, 142; Rusty Blackbird, 4; Pine Grosbeak, 1; Common Redpoll, 448; Snow Bunting, 44. F. G. Bard, R. W. Nero, A. E. Swanston, R. W. Fyfe, F. Lahrman (compiler), E. Fox.

DILKE, December 25, $25 \mathrm{mi} .2 \mathrm{hrs}$. by car, temp. $10-20^{\circ}$ wind NW 5-15, sunny, snow in drifts with almost clear patches. Open prairie and farm land. 8 species, 640 individuals. Golden E a g le, 1; Sharp-tailed Grouse, 16; Gray (Hungarian) Partridge, 6; Black-billed Magpie, 13: Bohemian Waxwing, 25; House Sparrow, 18; Common Redpoll, 261; Snow Bunting 300. Add: Downy Woodpecker, 1, Dec. 31; Horned Lark, 1, Dec. 24.) - Mr. and Mrs. S. R. Belcher and Margaret and Boswell Belcher.

GRENFELL, January 1， 1958，251/2 mi. by truck in $1 \frac{1}{4} \mathrm{hrs}$. and around farm yard during the day. Temp. -12 to $6^{\circ}$, clear, light wind, 4-5 inches snow. 7 species, 105 individuals. Hairy Woodpecker, 1; Black-billed Magpie, 16; Common Crow, 1; Blackcapped Chickadee, 2; House Sparrow. 50; Pine Grosbeak. 7; Common Redpoll, 28. (Add: Golden Eagle, 1, Dec. 30; Sharp-tailed Grouse, 1, Dec. 27; Bohemian Waxwing, 2, Dec. 24; Starling, 3, Jan. 2.) - Mr. and Mrs. J. Hubbard.

HiGH HILL, December $30,20 \mathrm{mi}$. and 16 party hrs. on foot. Temp. -20 to $-28^{\circ}$, wind $\mathrm{SW} 4$, clear and sunny, 10 inches of snow. 11 species, 65 individuals. Rough-legged Hawk, 1; Ruffed Grouse. 12; Hairy Woodpecker 2; Black-backed (Arctic) Three-toed Woodpecker, 1; Gray (Canada) Jay, 3; Blue Jay, 1; Black-billed Magpie, 4; Common Raven, 2; Black-capped Chickadee, 10; Pine Grosbeak, 6; Common Redpoll, 23. (Add: Downy Woodpecker, 1, Dec. 22; Snow Bunting, 20, Dec. 25.) - Anton and Steve Waycheshen.

LEADER, Dec. 30, 4 hrs. about the yard and on a short horseback ride. Temp. $4^{\circ}, 2$ inches of snow. 3 species, 22 individuals. Ring-necked Pheasant. 10; Black-billed Magpie, 8; Black-capped Chickadee, 4. - Dais.

\section{Meyers.}

McLEAN, Jan. 1, 1958; 2 mi., 3 hrs. on foot. Temp. $10^{\circ}$, clear and sunny, 5 inches of snow. 6 species,
40 individuals. Hairy Woodpecker, 2 ; Downy Woodpecker, 2; Black-capped Chickadee, 8; Waxwing, 12; Pine Grosbeak, 4; Common Redpoll, 12.Mrs. Newton and Mrs. Harold Bray. NAICAM, Jan. 1, $6 \mathrm{mi}$. by car and $5 \mathrm{mi}$. on foot in $5 \frac{1}{2} 2$ hours. Temp. $10^{\circ}$, wind light. sunny, 4 inches of snow. 8 species, 159 individuals. Ruffed Grouse, 2; Downy Woodpecker, 1 Black-billed Magpie, 1; Black-capped Chickadee, 4; House Sparrow, 12; Pine Grosbeak, 6; Common Redpoll, 125; Snow Bunting, 8. (Add: Sharptailed Grouse, 3; Hairy Woodpecker, 1; Starling,5.) - W. Yanchinski.

PRINCE ALBERT, Dec. 27, 41/2 mi on foot in $6 \frac{1 / 4}{4} \mathrm{hrs}$. Temp. $25^{\circ}$, wind East at 10,12 inches of snow. 13 species, 336 individuals. $\mathrm{P}$ i g e o $\mathrm{r}$ Hawk, 1; Downy Woodpecker, 1 Black-billed Magpie, 2; Common Raven, 9; Common Crow, 33; Blackcapped Chickadee, 25; Boreal (Brown. capped) Chickadee, 5; Brown Creeper, 4; House Sparrow, 36; Evenin€ Grosbeak, 76; Pine Grosbeak, 18 Hoary Redpoll, 1; Common Redpoll 125. (Add: Goshawk, 1 Dec. 22; Gray (Canada) Jay, 1. Dec. 23; Bohemiar Waxwing, 1, Dec. 22.) -Don Karasiul REGINA, Dec. 28, $177 \mathrm{mi}$. by ca and $22 \mathrm{mi}$. on foot, 44 party hour by car and 18 party hours on foot 9 observers in 4 parties. Temp -18 t $-10^{\circ}$, wind $\mathrm{E}$ to $\mathrm{SE} 8$ to $20 \mathrm{mph}$ 244 species, 1,747 individuals. Whist ling Swan, 1; Canada Goose, 87; Mal lard, 300; Pintail, 2; Canvasback, 1 Lesser Scaup, 11; Common Golden eye, 1; Ruddy Duck, 1; Sharp-tailer Grouse, 11: Gray (Hungarian) Par tridge, 25; Great Horned Owl, 1 Snowy Owl, 3; Hairy Woodpecker 1 ; Downy Woodpecker, 2; Black-bil led Magpie, 48; Common Crow, 2 Black-capped Chickadee, 39; Red breasted Nuthatch, 1; Golden-crown ed Kinglet, 1; Bohemian Waxwing 70; Northern Shrike, 1; House Spar row, 575; Common Redpoll, 133; Snor Bunting, 430. (Add: American Wid geon (Baldpate). Blue-winged Tea Redhead, Coot, Meadowlark, all see Dec. 21 and Jan. 1.)-F. Brazier, Mr W. K. Cruickshank, E. Fox (com piler), R. Fox, D. Gilroy, G. Leding ham, R. Nero, D. Poudrier, J. Roor SASKATOON, Dec. $26,22 \mathrm{mi}$. O foot and $133 \mathrm{mi}$. by car, 12 part hours on foot and $161 / 2$ party hour by car; 22 observers in 5 partie Temp. 16 to $34^{\circ}$, wind NE 20 to 3 
mph, partially cloudy, 2 inches snow. 15 species, 4,662 individuals. Miallard, 10; Sharp-tailed Grouse, 35; Gray (Hungarian) Partridge, 23; Ringnecked Pheasant, 4; Great Horned Owl, 2; Hairy Woodpecker, 5; Downy Woodpecker, 1; Black-billed Magpie, 107; Black-capped Chickadee, 29; Bohemian Waxwing, 1,550; House Sparrow, 1,206; Evening Grosbeak, 24; Pine Grosbeak, 58; Common Redpoll, 1,260; Snow Bunting, 348. J. S. MacLennan, Ted Wedge, Terry, Wendy and Lindy-Lou Wedge, R. Pravda, A. Grady, R. Gerrity, G. Gerrity, D. Morris, B. Mills, J. F. Roy (compiler), Dr. and Mrs. R. Bremner and Margaret Bremner, $R$. Folker, B. Gollop, J. Shadick, J. Hogg, J. Mackenzie, P. Cordrey. (Members of the Saskatoon Natural History Society).

KULL CREEK, Dec. 27, $14 \mathrm{mi}$. on foot and $19 \mathrm{mi}$. by car, in 20 party hours. Temp $16^{\circ}$, strong SE wind, inch of snow. 20 species, 1,092 individuals. Mallard, 24; Rough-legged Hawk, 1; Golden Eagle, 1; Ruffed Grouse, 1; Sharp-tailed Grouse, 120; Gray (Hungarian) Partridge, 12; Ring-necked Pheasant, 3; Great Horned Owl, 1; Snowy Owl, 1; Downy Woodpecker, 2; Black-billed Magpie, 7; Black-capped Chickadee, 33; Norhern Shrike, 1; House Sparrow, 246; Teadow Lark, 1; Evening Grosbeak, ; Pine Grosbeak, 30; Common Redoll, 500; Tree Sparrow, 2; Snow Bunting, 45. (Add: Horned Lark, 22, ec. 29; Bohemian Waxwing.)-Reid hearwood, Harry Williams, Kenny Vright, Peter, Carol and Berna wain, Mr. and Mrs. Robert Mann, Ir. Steve Mann (compiler), and tiss Helen Mann.

OMME, Dec. $22,6 \mathrm{mi}$. by foot in hours. Temp $0^{\circ}$, wind 5 mph., 12 ches of snow. 8 species, 106 inividuals. Goshawk, 1; Sharp-tailed rouse, 20; Snowy Owl, 1; Blackilled Magpie, 1; Common Raven, 7; ouse Sparrow, 25; Pine Grosbeak, Snow Bunting, 50. (Add: Ruffed rouse, 1, Dec. 24; Black-capped hickadee, 1, Dec. 24; Hoary Redll, 1, Jan. 1.)-Donald and Ronald ooper.

PEERS, Jan, 3, $34 \mathrm{mi}$. by car and $\mathrm{mi}$. on foot, $2 \frac{1}{4} \mathrm{hrs}$. by car, and $1 / 4$ hrs. on foot. Temp. $14^{\circ}$, clear, ind light, 3-4 inches of snow. 6 ecies, 475 individuals. Snowy Owl, Black-billed Magpie, 4; Black- capped Chickadee, 1; House Sparrow, 50; Pine Grosbeak, 19; Common Redpoll, 400. (Add: Ruffed Grouse, 2. Dec. 29; Sharp-tailed Grouse, 8, Dec. 30; Great Horned Owl, 1, Dec. 29.)-Stuart Langley.

SPIRIT LAKE, Dec. 29, $10 \mathrm{mi}$. on foot in $8 \frac{1}{2}$ party hours. Temp. $25^{\circ}$ clear, 8-10 inches of snow. 10 species, 143 individuals. Ruffed Grouse 1; Great Horned Owl, 3; Hairy Woodpecker, 9; Downy Woodpecker, 8; Black-billed Magpie, 4; Black-capped Chickadee, 70; House Sparrow, 30; Pine Grosbeak, 1; Common Redpoll, 12; Snow Bunting, 5. (Add: Goshawk, 1, Dec. 30; Sharp-tailed Grouse, 1, Dec. 30; Bohemian Waxwing, 2, Dec. 22; Evening Grosbeak, 3. Dec. 30; Hoary Redpoll, 1, Dec. 23.)-Joyce Gunn, Bill Anaka.

TISDALE, Jan. 1, 2 hrs. on foot about the area of Tisdale adjacent to the Doghide Creek. Temp. 0 to $10^{\circ}$, partly sunny, 4 inches of snow. 11 species, 84 individuals. Ruffed Grouse, 1; Hairy Woodpecker, 1; Downy Woodpecker, 1; Black billed Magpie, 5; Common Raven, 1; Black-capped Chickadee, 5; Bohemian Waxwing, 15; Starling, 2; House Sparrow, 15; Evening Grosbeak, 30; Pine Grosbeak. 8. - K. Elwin Baines.

WYNYARD, Dec. 25,8 species, 55 indivduals. Ruffed Grouse, 3; Hairy Woodpecker, 2; Downy Woodpecker, 2; Black-billed Magpie, 1; Blackcapped Chickadee, 12 ; Northern Shrike, 1; House Sparrow, 28; Common Redpoll, 6. (Add: Great Horned Owl, 3, Dec. 22; Waxwing, 4, Dec. 30; Evening Grosbeak, 4, Dec. 23.) D. Bardal.

YORKTON, Dec. $26,12 \frac{1}{2} \mathrm{mi}$. on foot, $110 \mathrm{mi}$. by car, 81/2 party h.rs. on foot, $19 \frac{1 / 2}{2}$ party hrs. by car, 17 observers in 6 parties. Temp. 19 to $23^{\circ}$, wind SE at $30 \mathrm{mph}$., overcast, 6 inches of snow. 15 species, 894 individuals. Ruffed Grouse, 1; Sharptailed Grouse, 18; Gray (Hungarian) Partridge, 36; Great Horned Owl, 2; Downy Woodpecker, 2; Blue Jay, 2; Black-billed Magpie, 28; Black-capped Chickadee, 9; Bohemian Waxwing, 41; Starling, 4; House Sparrow, 219; Pine Grosbeak, 25; Hoary Redpoll, 2; Common Redpoll, 231; Snow Bunting, 274. (Add: Snowy Owl, 1, Dec. 29; Hairy Woodpecker, 1, Dec. 24.)-Brother Eugene, Brother Joseph, Henry Chilman, Jr., Tom Cursons, Archie Fraser, Art Gellert, Billy 
Horseman, Dr. and Mrs. Stuart Houston (compilers), Ronald McDonald, Preston McDonald, Bob Pearce, Wayne Pearce, Elwood Sharpe, Cliff Shaw, Jeff Smith, Donald Swaby. (Yorkton Natural History Society).

Ed. note: The following report came in too late to be totalled with the above reports.

FORT SAN, Dec. 22, $28 \mathrm{mi}$. by car in $10 \mathrm{mi}$. area, $3 \frac{1 / 4}{\mathrm{hrs}} 9$ species, 208 individuals. Golden Eagle, 1;
Downy Woodpecker, 3; Black-bille Magpie, 7; Black-capped Chickade 12; Northern Shrike (?); House Spa row, 70; Evening Grosbeak, 3; Rec poll, 110; Snow Bunting, 1. (Ad C o m m o n Merganser, Ruffe Grouse, Hairy Woodpecker, Blue Ja White-breasted Nuthatch, Bohemic Waxwing, Pine Grosbeak.) - Jad Lowe, Errol Cochrane, John Lenno E. M. Callin.

\section{Goshawk Trapping in Alberta}

\section{By A. F. Oeming, President, Edmonton Zoological Society}

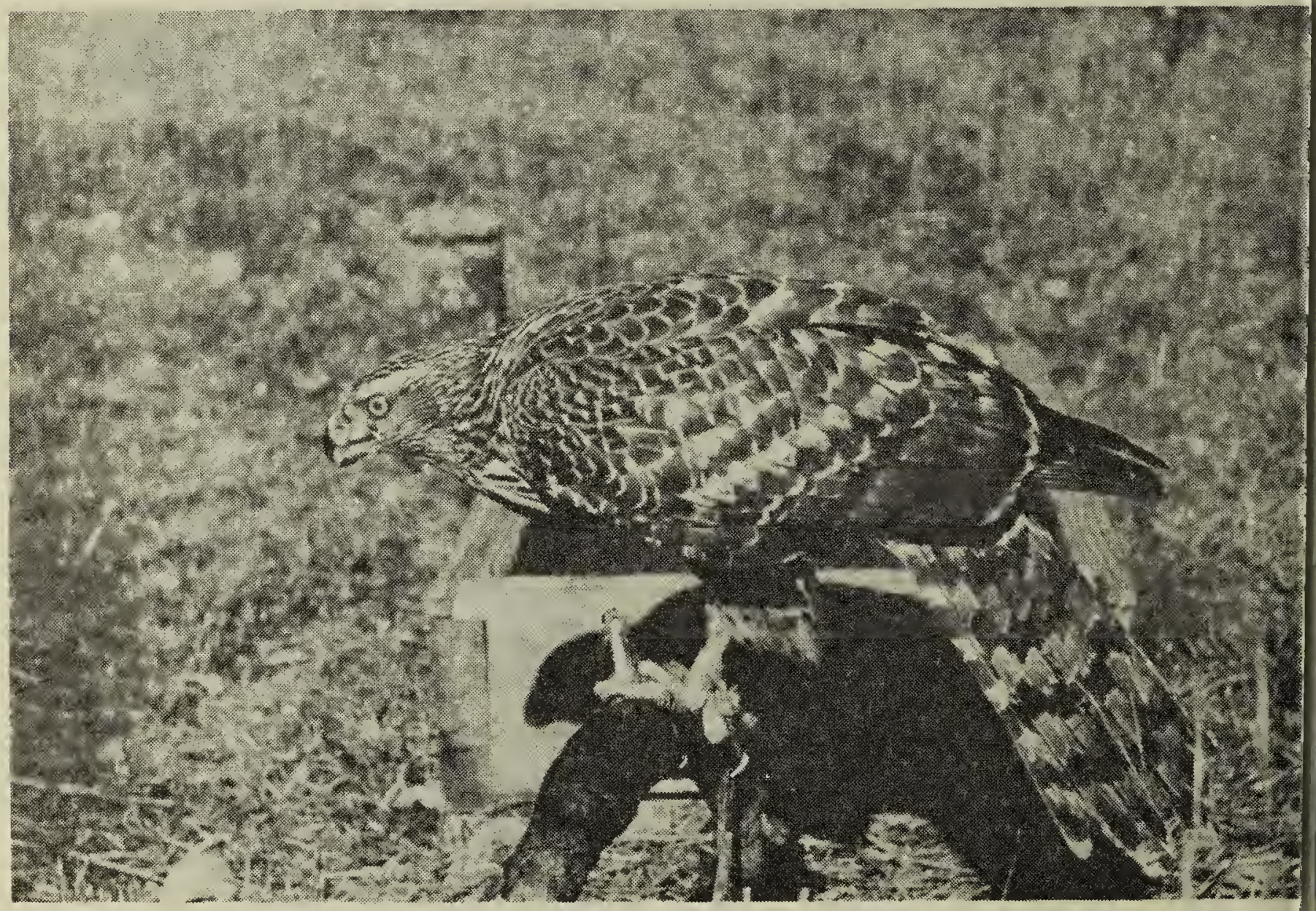

Photo by Heinz Meng

Immature female Goshawk on bow perch.

My interest in the live trapping of the Goshawk, Astur atricapillus, was prompted by an active interest in the art of falconry and a desire to band a number of these not too common raptors. From 1949 to 1951 I took a small number of birds, using such laborious trapping methods as the hand bow-net with tethered pigeons. Not until the year 1952, and after a considerable number of trapping techniques had been experimented with, did I manage to devise a fool-proof method that would allow for a worthwhile number of Goshawks to be captured each season. This was through the use of the
Swedish Goshawk trap (Figure of a pattern similar to those used the gamekeepers of Sweden. T trap has the advantage of not quiring constant supervision. Ot traps used, such as the autom: bow-net and various German mal invariably cramped the captive $b$ so that in its struggles in the $t$ much of its plumage was dama A bird with any part of its plum damaged is of no value to a falcor The Swedish trap, when well net allows the newly captured Goshar to move from end to end freely with no feather damage. The Sw ish trap is simple to build and of 